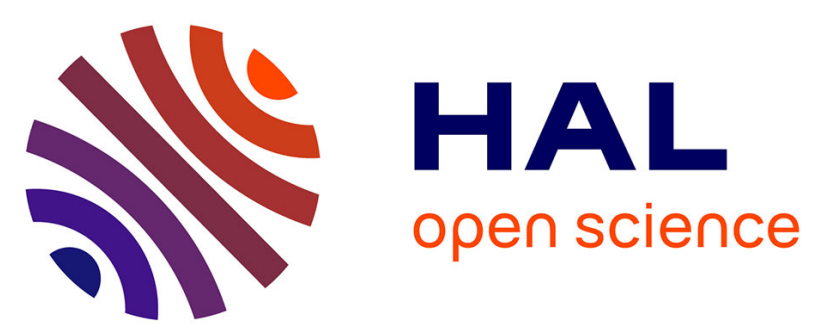

\title{
Absence of ABCG2-mediated mucosal detoxification in patients with active inflammatory bowel disease is due to impeded protein folding
}

J. Jasper Deuring, Colin de Haar, Chantal L. Koelewijn, Ernst J. Kuipers, Maikel Peppelenbosch, C. Janneke van Der Woude

\section{To cite this version:}

J. Jasper Deuring, Colin de Haar, Chantal L. Koelewijn, Ernst J. Kuipers, Maikel Peppelenbosch, et al.. Absence of ABCG2-mediated mucosal detoxification in patients with active inflammatory bowel disease is due to impeded protein folding. Biochemical Journal, 2011, 441 (1), pp.87-93. 10.1042/BJ20111281 . hal-00658163

\section{HAL Id: hal-00658163 https://hal.science/hal-00658163}

Submitted on 10 Jan 2012

HAL is a multi-disciplinary open access archive for the deposit and dissemination of scientific research documents, whether they are published or not. The documents may come from teaching and research institutions in France or abroad, or from public or private research centers.
L'archive ouverte pluridisciplinaire HAL, est destinée au dépôt et à la diffusion de documents scientifiques de niveau recherche, publiés ou non, émanant des établissements d'enseignement et de recherche français ou étrangers, des laboratoires publics ou privés. 


\title{
Absence of ABCG2-mediated mucosal detoxification in patients with active inflammatory bowel disease is due to impeded protein folding
}

\author{
J. Jasper Deuring ${ }^{1}$, Colin de Haar ${ }^{1}$, Chantal L. Koelewijn ${ }^{1}$, Ernst J. Kuipers ${ }^{1}$, Maikel P. \\ Peppelenbosch ${ }^{1}$ and C. Janneke van der Woude ${ }^{1}$ \\ Erasmus MC University Medical Centre Rotterdam, Department Gastroenterology and Hepatology ${ }^{1}$, \\ 3015 CE Rotterdam, The Netherlands
}

Correspondence:

J. Jasper Deuring

Department of Gastroenterology and Hepatology

Erasmus MC Rotterdam

's-Gravendijkwal 230

3015 CE Rotterdam

The Netherlands

Phone: 0031-10-7038695

Fax: 0031-10-7032793

E-mail: j.deuring@erasmusmc.n!

Author contribution: JJD, designed and performed research, write manuscript; $\mathrm{CdH}$, analysed data, extensive drafting of manuscript and study supervision; CLK, helped designing research; EJK, critical revision manuscript; MPP, critical revision manuscript; CJvdW, critical revision manuscript and study supervision.

Ethics: All authors declare that this study is conducted with the approval of the Erasmus MC University Medical Centre, Rotterdam, ethics committee.

Abbreviations: IBD, inflammatory bowel disease, CD, Crohn's disease, UC, ulcerative colitis, IEC, intestinal epithelium cells, NO, nitric oxide, ER, endoplasmic reticulum, PDI, protein-disulfide isomerase, GFP, green fluorescent protein, RFP, red fluorescent protein, 293t-AB, HEK293t cells transiently transfected with GFP-ABCG2, FFPE, formalin fixed paraffin embedded, IHC, immunohistochemistry. 


\begin{abstract}
Xenotoxic damage in inflammatory diseases, including inflammatory bowel disease (IBD), is importantly compounded by reduced activity of the xenobiotic transporter ABCG2 during the inflammatory state. An association between activation of the unfolded protein response pathway and inflammation prompted us to investigate the possibility that reduced ABCG2 activity is causally linked to this response. To this end we correlated expression of ABCG2 and the unfolded protein response marker GRP78 in colon biopsies from healthy individuals $(n=9)$, and patients with inactive $(n=67)$, or active $(n=55)$ IBD, ischemic colitis $(n=10)$, or infectious colitis $(n=14)$. In addition, tissue-specimens throughout the small bowel from healthy individuals $(n=27)$, and from patients with inactive $(n=9)$ or active $(n=25)$ Crohn's disease were co-stained for ABCG2 and GRP78. In all biopsies from patients with active inflammation, irrespective of the underlying disease, an absolute negative correlation is observed between epithelial ABCG2 expression and GRP78 expression, suggesting that inflammation-dependent activation of the unfolded protein response is responsible for suppression of ABCG2 function. The link between the unfolded protein response and functional ABCG2 expression is further corroborated by live-imaging of ABCG2 expressing cells which show that various inflammatory mediators, including nitric oxide, activate the unfolded protein response and concomitantly reduce plasma membrane localisation as well as transport function of ABCG2. Thus a novel mechanism for explaining xenobiotic stress during inflammation emerges, in which intestinal inflammation actives the unfolded protein response in turn abrogating defences against xenobiotic challenge by impairing ABCG2 expression and function.
\end{abstract}




\section{Introduction}

2

The intestinal epithelium is a single-cell physical barrier between the gut luminal content and mucosa, important for detection [1] and defence [2-3] against microbes. Intestinal epithelial cells (IEC) are constantly exposed to a large variety xenobiotic substances, including microbes and their products as well as exogenous (e.g. drugs or food) and endogenous (e.g. bile acids) toxic substances present in the luminal content. IEC utilize various mechanisms to maintain epithelial integrity in face of the xenobiotic challenge. They are protected against exogenous and endogenous toxic compounds by, among others, proteins from the ATP-binding cassette $(A B C)$ transporter family. This family includes the P-glycoprotein/Multidrug Resistance (P-gp/MDR1/ABCB1), Multidrug Resistance-associated Protein-1 (MRP1/ABCC1), Multidrug Resistance-associated Protein-2 (MRP2/ABCC2) and Breast Cancer Resistance Protein (BCRP/ABCG2) [4-5]. Especially the homodimeric xenobiotic transporter ABCG2, which specifically localises to the IEC apical membrane, is widely considered to be especially important in combating luminal-derived xenobiotic stress [6] [7].

Disruption of IEC integrity leads to inflammatory lesions, which are a hallmark of active inflammation in patients with inflammatory bowel disease (IBD). Appropriate response to such disruption requires rapid mucosal healing by cell division, differentiation and maturation to restore barrier function [8-9]. Active inflammation in patients with IBD has been associated with dramatically reduced expression of $A B C$-transporters in IEC and the resulting diminished capacity to deal with luminal xenobiotic challenge is thought to interfere with the efficiency of regenerative responses [7, 10-12]. Further compounding this problem is that several medications used in IBD are specific substrates of ABCG2. Hence understanding of the mechanisms governing ABCG2 expression is highly relevant for devising improved therapy [13-14]. Nevertheless, the molecular mechanisms driving ABCG2 expression during inflammatory responses remain obscure at best.

Generally, excreted and membrane-bound proteins are translated in the endoplasmic reticulum (ER). If ER function is disrupted, proteins can accumulate inside the ER and this can initiate a specific ER stress syndrome denoted the unfolded protein response. Recently, abnormalities in the ER stress response pathways have been associated with the pathogenesis of IBD [15-17]. Proper function of the ABCG2 protein depends on Nglycosylation and the formation of inter/intra molecular disulfide bonds [18-19]. These bonds are formed inside the ER by protein-disulfide isomerases (PDI). Inflammatory conditions, such as increased nitric oxide production, can dramatically reduce the efficiency of these PDI, which can initiate misfolding of various proteins leading to ER stress [20-21]. As correct ABCG2-protein folding is necessary for its functionality, we hypothesized that mucosal inflammation in the setting of IBD alters proper ABCG2-protein folding and thereby reduces its apical expression. In this study we describe a novel mechanism by which ABCG2 protein expression is decreased during active inflammation. 


\title{
Experimental procedures
}

2

3

4

5

6

7

8

\begin{abstract}
Patient materials
Formalin-fixed paraffin-embedded (FFPE) colonic biopsies were collected from nine healthy individuals and from 76 patients: 25 with Crohn's disease (CD), 36 with ulcerative colitis (UC), 10 with ischemic colitis and 5 with infectious colitis. Biopsies throughout the small intestine were collected from 17 other healthy individuals and 34 CD patients. For mRNA isolation freshly-frozen biopsies were collected from six healthy individuals and from 15 patients: 8 with inactive IBD and 7 with active IBD.

The demographic characteristics, number of biopsies per group, duration of disease and medication use are described in supporting Tables 1,2 and 3 (included in the supporting section).
\end{abstract}

\section{Immunohistochemistry and scoring}

The FFPE sections were immunohistologically stained using the primary antibodies BXP21 anti-ABCG2 (Santa Cruz Biotechnology, Santa Cruz, CA) and BiP anti-GRP78 (Cell Signalling, Boston, USA) according to the manufacturers' protocol (included in the supporting section). The expression of ABCG2 and GRP78 in IEC was scored by intensity over the whole slide by two independent observers, and discrepancies were re-assessed to come to a final agreement. Individual scores were used for statistical analyses, whereas they were combined into three subgroups for graphic representation. For ABCG2 positive staining at the apical membrane of the epithelial celis was defined as positive if $>50 \%$ of the IEC were positive, mild $15-50 \%$, or negative $<15 \%$. Immunoreactivity for GRP78 was also defined in terms of positive IEC, but now the percentage were $>70 \%$ defined as positive, $20-70 \%$ mild, $<20 \%$ negative.

mRNA isolation and quantitative PCR

mRNA was isolated from colonic biopsies, which were taken from CD and UC patients, using the NucleoSpin Extract II mRNA isolation kit (Macherey Nagel, Düren, Germany). Gene expression of GapdH, IL8, IL 1beta, ABCG2, villin, and ER stress associated gene CCAAT/enhancer binding protein (C/EBP) homologous protein (CHOP) [20] were measured using quantitative PCR (qPCR, IQ5, Bio-Rad). Villin and ABCG2 are both IEC specific, thus ABCG2 mRNA is corrected for Villin to prevent underestimation in biopsies with less IEC. A detailed protocol and the primer sequences involved are presented in the supporting section.

\section{Cell culture}

HEK293t (ATCC\# CRL-1573) cells were cultured according to routine procedures. Transient transfection was performed using Polyethylenimine (PEI, Polysciences, Eppelheim, Germany). S-nitroso-N-acetylpenicillamine (SNAP, Sigma-Aldrich) was used as a NO donor. Tunicamycin from Streptomyces sp. (Sigma-Aldrich) was used as a positive control for ER stress induction. The cell culture supernatant nitrate concentrations were measured using Griess Reagents (Cayman Chemical, Michigan, USA) according to the manufacturers protocol. Additional cell culture methods are described in the supporting section.

XBP1 splicing

ER stress and unfolded protein response activity was assessed by identify mature XBP1 mRNA and its spliced variant [16] (included in the supporting section). 


\section{Construct cloning}

2 To investigate ABCG2 functionality we created a green fluorescent protein (GFP)-ABCG2

3 fusion construct (included in the supporting section).

4

\section{Fluorescent microscopy}

The GFP-tagged proteins were examined using a confocal microscope (LSM510META, Zeiss). For the transport assay, nuclear Hoechst 33342 (4 $\mu \mathrm{M}$, Sigma-Aldrich) accumulation was determined at $30 \mathrm{~s}$ intervals during a total of $12 \mathrm{~min}$. The ER was stained with ER-RFP (Red Fluorescent Protein) using ER-Tracker (Molecular Probes, Paisley, UK) according to the manufacturer's instruction. We analyzed the GFP tagged proteins with a $488 \mathrm{~nm}$ laser, Hoechst with a $405 \mathrm{~nm}$ laser and the ER-RFP with $543 \mathrm{~nm}$ laser. A specific ABCG2 transport inhibitor Ko143 (2 $\mu \mathrm{M}$, Sigma-Aidrich) was used to indentify $A B C G 2$ transport specificity.

\section{SDS-page and fluorescent immunoblotting}

For checking correct length of fusion proteins, whole cell lysates were analysed on immunoblot from standard SDS-page gel, using antibodies against ABCG2 (BXP-21, Santa Cruz Biotechnology) and GFP (polyclonal, rabbit IgG, Invitrogen) (included in the supporting section).

\section{Statistics and software}

Statistical analyses were performed using the SPSS 11.0 software package for Windows. Data on different patient groups were compared using the Mann-Witney $U$ test with Kruskal-Wallis post test. Using univariate and multivariate multinomial logistic regression analysis, we examined the association between colitis and GRP78 and ABCG2 expression, adjusting for age and duration of disease. A two-tailed $p$ value $<0.05$ was accepted as statistically significant. Images were composed using Adobe Photoshop CS3. 


\section{Results}

2

3

4

5

6

Colonic inflammation reduces apical ABCG2 expression in IEC

To investigate ABCG2 expression during human intestinal inflammation, ABCG2 expression in colonic biopsies was qualitatively assessed employing immunohistochemistry (IHC). As expected IEC in biopsies of control subjects as well as of IBD patients in remission (with no active inflammation) show strong specific staining for ABCG2 at the apical surface (Figure 1A). In contrast, apical IEC ABCG2 staining is dramatically reduced in patients with active IBD, in the colon as well as in the small intestine, and also in biopsies from ischemic colitis and infectious colitis patients (Figure $1 \mathrm{~A})$. Thus diverse colonic inflammatory responses are uniformly characterised by down regulation of apical $A B C G 2$ levels, suggesting that processes common to these alternative modes of inflammation underlie the absence of apical ABCG2 intestinal inflammation.

In apparent agreement, quantitative study of ABCG2 levels in the human colon reveals significantly less ABCG2 expression in active colitis (24\% positive expression, 13/54), ischemic colitis (10\% positive staining, $1 / 10)$ and infectious colitis $(0 \%$ positive staining $0 / 14)$ as compared to either inactive colitis ( $80 \%$ positive expression, $53 / 67)$ or control tissues (100\% positive expression, 9/9), all $p<0.001$ (see Figure 1B). There is no significant difference in ABCG2 levels between active CD $(27 \%, 6 / 22)$ vs. active UC $(22 \%$, $7 / 32)$ and inactive CD $(67 \%, 18 / 27)$ vs. inactive UC $(88 \%, 35 / 40)$. As a control, we examined left- and right-sided biopsies from IBD patients and controls to investigate a confounding possible differential expression of $A B C G 2$ based on position in the colon, but this analysis demonstrates that only the degree of inflammation is an important factor guiding apical ABCG2 levels. Thus inflammation per se is associated with a marked reduction of apical $A B C G 2$ expression in the human colon.

Small bowel inflammation is associated with down regulation of apical ABCG2 expression Mirroring the findings obtained in the human colon, small bowel biopsy specimens obtained from CD patients with active disease by double balloon endoscopy exhibit markedly less ABCG2 staining when compared to biopsies obtained from patients with inactive disease or no intestinal inflammatory phenomena (see Figure 1A). In active CD, there is significantly less small bowel ABCG2 staining ( $75 \%$ negative staining, 19/25) as compared to inactive CD $(11 \%$ negative staining, $1 / 9)$ and controls $(8 \%$ negative staining, $2 / 25$ ), both $\mathrm{P}<0.05$ (see Figure $1 \mathrm{C}$ ). We find no significant differences between inactive $\mathrm{CD}$ and controls. In all cases current drug use did not influence the expression of ABCG2. We conclude that human intestinal inflammation is accompanied by reduced expression of xenobiotic transporter $A B C G 2$ and experiments were initiated to identify the underlying molecular mechanisms.

Intestinal inflammation acts to reduce ABCG2 via a post-transcriptional mechanism To delineate the molecular events reducing the capacity of the intestine to cope with xenobiotic challenge we first determined whether genomic or post-transcriptional processes are involved in this effect. To this end, we measured ABCG2 mRNA levels in freshly frozen colon biopsies from healthy individuals, inactive and active IBD patients. Importantly, ABCG2 mRNA, levels in IEC, do not differ between these three groups (all $p>0.8$ ). The expression, however, of the pro-inflammatory cytokine IL8 and IL1beta are 10and 8 -fold higher $(p<0.05)$ in biopsies with active mucosal inflammation (Figure 2 ) and thus technical issues do not underlie the absence of intestinal inflammation-induced effects on apical ABCG2 expression. Hence we are forced to conclude that down modulation of ABCG2 in human intestinal inflammation is brought about by a posttranscriptional process. 


\section{Activation of the protein unfolded protein response accompanies human intestinal inflammation}

As it is becoming increasingly clear that activation of the unfolded protein response accompanies inflammatory processes and that ER stress may interfere with proper production of complexly folded proteins (the "half" transporter ABCG2 transverses the membrane 6 times), we examined whether the ER stress pathway is activated in intestinal inflammation and to which extent this activation mirrors the down regulation of ABCG2. To this end we employ the expression of the bona fide ER stress marker GRP78. Representative images of the GRP78 immunohistochemical analyses of the same biopsies as stained for ABCG2 are shown in Figure 3A. There is a significant increase in GRP78 expression in active colitis (32\% positive expression, $16 / 54)$, ischemic colitis $(90 \%$ positive staining, $9 / 10)$ and infectious colitis (79\% positive staining $11 / 14)$, compared to inactive colitis $(0 \%$ positive expression, $0 / 67)$ and control tissues $(0 \%$ positive expression, $0 / 9$ ), all $p<0.001$ (see Figure 3B). There are no significant differences in GRP78 expression between active $C D$ and active $U C$ or between inactive $C D$ and inactive UC. Also there are no differences in GRP78 expression in left- and right-sided colon biopsies taken from the same patient. We conclude that colonic inflammation is accompanied by ER stress response which is absent in the un-inflamed colon.

In the small bowel biopsies of active CD patients there is increased GRP78 expression (29\% positive expression, 6/21) compared to inactive CD patients $(0 \%$ positive staining, $0 / 9$ ) and controls ( $8 \%$ positive expression, $2 / 25) p<0.05$ (see Figures $3 A$ and $3 C$ ). In all actively inflamed tissue samples elevated GRP78 expression is evident, irrespective of disease type or location. ER stress during intestinal inflammation is further confirmed measuring the mRNA expression of ER stress associated gene, CHOP, in colonic biopsies. In biopsies taken from actively inflamed mucosa a significant increase in CHOP mRNA expression was measured, $p<0.01$ (see Figure 2B). Moreover, an increased ratio of spliced XBP1 mRNA was detected in patients with active intestinal inflammation (Figure 2C). We conclude that activation of the unfolded protein response and intestinal inflammation are highly associated processes and we initiated experiments to investigate whether ER stress explains down regulation of ABCG2 in intestinal inflammation.

\section{Impaired ABCG2 function in ER stressed cells}

To investigate the effect of activated ER stress on ABCG2 protein expression and function, we created a GFP-ABCG2 fusion protein. To this end, ABCG2 CDNA was cloned into a pEGFP-C1 vector which led to the production of GFP-ABCG2 after transfection (Figure 4A). The NO-donor SNAP was used to ascertain whether NO was able to induce ER stress in GFP-ABCG2 transfected HEK293t (293t-AB) cells. The Griess assay reveals SNAP application induces robust increases in $\mathrm{NO}_{2}$ concentration, a good proxy measure for NO production (see Figure 4B). The validity of assay is demonstrated by experiments showing an increase in the ratio of unspliced XBP1 mRNA over spliced XBP1 mRNA nine h after application of $0.625 \mathrm{mM}$ SNAP (see Figure 4C). In agreement administration of tunicamycin, a well established ER stress response inducer, increases the XBP1 splicing ratio to approximately the same level as SNAP application (supporting Figure 1B). Consistent with high ABCG2 transport activity, administration of Hoechst 33342 does not stain the nuclei of the 293t-AB cells (Figure 5A), also demonstrating the functionality of the ABCG2-GFP fusion protein in xenobiotic transport. Nevertheless, $16 \mathrm{~h}$ after addition of the ER-stress inducers SNAP or tunicamycin, the nuclei of 293t-AB cells become Hoechst 33342 positive (Figure 5A and supporting movies 1, 2 and 3). As shown in Figure 5B Hoechst 33342 also stains the nucleus blue in 293t-AB transfected cells in the presence of a specific ABCG2 inhibitor Ko143 (see also supporting movie 4). No altered GFP-ABCG2 protein function is found when SNAP or tunicamycin is added $48 \mathrm{~h}$ after transfection (see 
supporting Figure 1C). Hence, in this over expressing model system ER stress provokes a very strong decrease in ABCG2 function, in agreement with a scheme in which inflammation-dependent induction of ER stress is causatively related to a reduced capacity to deal with xenobiotic challenge.

5 In support for such a model, all images from 293t-AB cells with ER stress reveal reduced 6 apical membrane expression of GFP-ABCG2, with remaining intracellular expression. In 7 order to ascertain whether GFP-ABCG2 remains in the ER after stress induction, we 8 stained the ER with ER-RFP. When ER stress was present some GFP-ABCG2 co9 localized with ER-RFP, whereas the majority was present in other cellular compartments 10 (Figure 5C). As shown in supporting Figure 2 the localization and expression of non-ER 11 dependent GFP-tagged proteins (GFP-betaCatenin, GFP-H2B) was not changed during 12 ER stressed conditions. Thus protein folding difficulties seems incompatible with functional 13 expression of ABCG2. 


\section{Discussion}

2

The data presented in the current study are a consistent with a model in which intestinal inflammation drives an epithelial misfolded protein response, which in turn interferes with the ABCG2 expression and function leading to a diminished capacity of the epithelial compartment to cope with xenobiotic stress. Colonic and small bowel biopsies from IBD patients with active disease and those from patients with ischemic and infectious colitis all show reduced apical ABCG2 expression in IEC compared to controls and IBD patients in remission. The decline of ABCG2 expression in the presence of active inflammation in IBD patients corresponds with the findings of two studies done exclusively on UC patients [7, 22]. In addition, we found that ABCG2 expression is not affected by medication use, patient age, or location of the inflammation.

Expression of $A B C G 2$, like that of many other proteins, can be regulated at various levels. In addition to a decline in ABCG2 protein expression, others have described a reduction in ABCG2 mRNA expression in actively inflamed intestine [7, 22]. We corrected the ABCG2 mRNA expression for Villin to prevent underestimation of ABCG2 expression, since both genes are specific for IEC. Our results show no reduction of ABCG2 mRNA expression in biopsies with active mucosal inflammation. However, since the sample size used here is smaller than those in previous studies, we cannot completely rule out the contribution of reduced mRNA expression to the decline in ABCG2 protein expression. As such our findings merely imply that the decline in mRNA expression probably does not solely account for the complete loss of ABCG2 protein expression discernable on our IHC images and that post-transcriptional mechanisms must be involved. The observation that unfolded protein responses are both a general and specific to intestinal inflammation suggest a causal link between ER stress and diminished apical ABCG2 levels. Indeed, ABCG2 is a "half" transporter, 6 times membrane spanning protein, functionally critically dependent on homodimerisation, $\mathrm{N}$-linked glycosylation and various disulfide bonds and is thus is a likely early victim of ER stress responses [18-19]. Disrupted protein-folding mechanisms that can lead to ER stress during active inflammation have recently been linked to IBD [16, 23-24] and may weil involve reduced capacity of the IEC compartment to deal with the xenobiotic stress and thus in turn interfere with demanding regenerative responses that an adequate response to IBD-related intestinal challenges requires.

Our inflammation links to unfolded protein responses remains unclear, but associated with inflammatory responses is an excessive production of NO, which in turn is predicted to disturb the function of redox-state sensitive disulfide-bonding chaperones like PDI that are necessary for proper protein folding [25]. In all biopsies with active inflammation (i.e. IBD and non-IBD related), the GRP78 expression is significantly higher than in the biopsies of IBD patients in remission, and in those of healthy controls. Furthermore, expression of CHOP mRNA (an ER stress marker) is increased in biopsies with active mucosal inflammation. In addition to this, biopsies with active inflammation reveal an increased ration of spliced XBP1 mRNA expression. Thus factors common to multiple types of inflammation must be involved here and as NO production is one of the few cellular responses described to generally accompany inflammation, it is tempting to attribute a important role to excessive NO generation in inflammation-related induction of ER stress responses [23, 25-27]. Indeed, we observe elevated XBP1 splicing [16] following application of the NO-donor, suggesting that NO can directly affect protein-folding mechanisms [20] and induce ER stress. In the current study we show that the reduction in $A B C G 2$ expression is inflammation-associated, which fits with the observations that ABCG2 expression is reduced in IBD-unrelated inflammatory conditions in other organs $[10,12,28]$. Other IBD associated proteins are also reduced in protein expression during active intestinal inflammation [29-31]. Further research is needed to investigate the role 
impeded protein folding and the effect of this on cellular processes. Especially, ABCG2mediated efflux of toxic components is thought to protect the epithelium of the gut and the underlying tissue to xenobiotic challenge. Importantly, as xenobiotic stress is directly linked to genotoxicity our results may also help explaining the still poorly understood relation between intestinal inflammation and colorectal cancer [32]. Decreased ABCG2 expression may have other deleterious consequences as well. Sulfasalazine, a derivate of mesalazine (5-ASA) that is commonly used in the treatment of IBD patients, is actively excluded from the epithelial compartment by ABCG2 [33]. Rodents with reduced IEC ABCG2 expression have increased 5-ASA trough levels [34]. In addition to its anti-

10 inflammatory effect, accumulation of sulfasalazine can be toxic for epithelial cells [35-36].

11 Thus reduced expression and function of ABCG2 may therefore greatly enhance the 12 sensitivity of IEC to sulfasalazine and limit the use of this compound for the treatment of IBD. Hence understanding the mechanisms governing ABCG2 expression are highly relevant for efforts with respect to rational design of novel therapy

In conclusion, IEC ABCG2 protein expression is decreased in the inflamed bowel probably as a result of incorrect protein folding due to inflammation-driven ER dysfunction. Thus a

17 novel paradigm explaining xenobiotic stress during inflammation emerges, in which 18 intestinal inflammation actives the unfolded protein response in turn abrogating 19 defences against xenobiotic challenge by impairing ABCG2 expression and function. 


\section{Acknowledgements}

2

3 The authors thank Dr. R. Smits for providing the GFB-betaCatenin and GFP-H2B fusion 4 constructs. The authors also thank Dr. H. van Dekken and Dr. K. Biermann for 5 pathological assessment of the intestinal cross sections. 


\section{References}

21 Abreu, M. T. (2010) Toll-like receptor signalling in the intestinal epithelium: how

3 bacterial recognition shapes intestinal function. Nat. Rev. Immunol. 10, 131-144

42 Perminow, G., Beisner, J., Koslowski, M., Lyckander, L. G., Stange, E., Vatn, M. H.

5 and Wehkamp, J. (2010) Defective paneth cell-mediated host defense in pediatric ileal

6 Crohn's disease. Am. J. Gastroenterol. 105, 452-459

73 Salzman, N. H., Underwood, M. A. and Bevins, C. L. (2007) Paneth cells,

8 defensins, and the commensal microbiota: a hypothesis on intimate interplay at the

9 intestinal mucosa. Semin. Immunol. 19, 70-83

104 Dietrich, C. G., Geier, A. and Oude Elferink, R. P. (2003) ABC of oral

11 bioavailability: transporters as gatekeepers in the gut. Gut. 52, 1788-1795

125 Leslie, E. M., Deeley, R. G. and Cole, S. P. (2005) Multidrug resistance proteins:

13 role of P-glycoprotein, MRP1, MRP2, and BCRP (ABCG2) in tissue defense. Toxicol. Appl.

14 Pharmacol. 204, 216-237

156 Robey, R. W., To, K. K., Polgar, O., Dohse, M., Fetsch, P., Dean, M. and Bates, S.

16 E. (2009) ABCG2: a perspective. Adv. Drug Deliv. Rev. 61, 3-13

177 Englund, G., Jacobson, A., Rorsman, F., Artursson, P., Kindmark, A. and

18 Ronnblom, A. (2007) Efflux transporters in ulcerative colitis: decreased expression of

19

20

21

22

23

24

25

26

27

28

29

30

31

32

33

34

35

36

37

38

39

40

41

42

43 BCRP (ABCG2) and Pgp (ABCB1). Inflamm. Bowel Dis 13, 291-297

8 Dignass, A. U. (2001) Mechanisms and modulation of intestinal epithelial repair. Inflamm. Bowel Dis. 7, 68-77

9 Podolsky, D. K. (1997) Healing the epithelium: solving the problem from two sides. J. Gastroenterol. 32, 122-126

10 Petrovic, V., Wang, J. H. and Piquette-Miller, M. (2008) Effect of endotoxin on the expression of placental drug transporters and glyburide disposition in pregnant rats. Drug Metab. Dispos.

11 Poller, B., Drewe, J., Krahenbuhl, S., Huwyler, J. and Gutmann, H. (2010)

Regulation of BCRP (ABCG2) and P-glycoprotein (ABCB1) by cytokines in a model of the human blood-brain barrier. Cell Mol. Neurobiol. 30, 63-70

12 Wang, J. S., Teng, S. and Piquette-Miller, M. (2006) Effect of maternal inflammation on the expression of ABCB1 and ABCG2 in placenta. Clin. Pharmacol. Therap. 79

13 Krishnamurthy, P. and Schuetz, J. D. (2006) Role of ABCG2/BCRP in biology and medicine. Annu. Rev. Pharmacol. Toxicol. 46, 381-410

14 Doyle, L. A., Yang, W., Abruzzo, L. V., Krogmann, T., Gao, Y., Rishi, A. K. and Ross, D. D. (1998) A multidrug resistance transporter from human MCF-7 breast cancer cells. Proc. Natl. Acad. Sci. U S A. 95, 15665-15670

15 Heazlewood, C. K., Cook, M. C., Eri, R., Price, G. R., Tauro, S. B., Taupin, D., Thornton, D. J., Png, C. W., Crockford, T. L., Cornall, R. J., Adams, R., Kato, M., Nelms, K. A., Hong, N. A., Florin, T. H., Goodnow, C. C. and McGuckin, M. A. (2008) Aberrant mucin assembly in mice causes endoplasmic reticulum stress and spontaneous inflammation resembling ulcerative colitis. PLoS Med. 5, e54

16 Kaser, A., Lee, A. H., Franke, A., Glickman, J. N., Zeissig, S., Tilg, H., Nieuwenhuis, E. E., Higgins, D. E., Schreiber, S., Glimcher, L. H. and Blumberg, R. S. (2008) XBP1 links ER stress to intestinal inflammation and confers genetic risk for human inflammatory bowel disease. Cell. 134, 743-756

17 Shkoda, A., Ruiz, P. A., Daniel, H., Kim, S. C., Rogler, G., Sartor, R. B. and Haller, D. (2007) Interleukin-10 blocked endoplasmic reticulum stress in intestinal epithelial cells: impact on chronic inflammation. Gastroenterology. 132, 190-207

5018 Nakagawa, H., Wakabayashi-Nakao, K., Tamura, A., Toyoda, Y., Koshiba, S. and

51 Ishikawa, T. (2009) Disruption of N-linked glycosylation enhances ubiquitin-mediated 
1 proteasomal degradation of the human ATP-binding cassette transporter ABCG2. Febs. J. 2 276, 7237-7252

319 Wakabayashi, K., Nakagawa, H., Tamura, A., Koshiba, S., Hoshijima, K., Komada, 4 M. and Ishikawa, T. (2007) Intramolecular disulfide bond is a critical check point 5 determining degradative fates of ATP-binding cassette $(A B C)$ transporter ABCG2 protein. 6 J. Biol. Chem. 282, 27841-27846

720 Oyadomari, S., Takeda, K., Takiguchi, M., Gotoh, T., Matsumoto, M., Wada, I.,

8 Akira, S., Araki, E. and Mori, M. (2001) Nitric oxide-induced apoptosis in pancreatic beta 9 cells is mediated by the endoplasmic reticulum stress pathway. Proc. Natl. Acad. Sci. U S

10 A. $98,10845-10850$

1121 Uehara, T., Nakamura, T., Yao, D., Shi, Z. Q., Gu, Z., Ma, Y., Masliah, E., Nomura, Y. and Lipton, S. A. (2006) S-nitrosylated protein-disulphide isomerase links protein misfolding to neurodegeneration. Nature. 441, 513-517 22 Gutmann, H., Hruz, P., Zimmermann, C., Straumann, A., Terracciano, L., Hammann, F., Lehmann, F., Beglinger, C. and Drewe, J. (2008) Breast Cancer Resistance Protein and P-Glycoprotein Expression in Patients with Newly Diagnosed and TherapyRefractory Ulcerative Colitis Compared with Healthy Controls. Digestion. 78, 154-162 23 Kolios, G., Valatas, V. and Ward, S. G. (2004) Nitric oxide in inflammatory bowel disease: a universal messenger in an unsolved puzzle. Immunology. 113, 427-437 24 McGuckin, M. A., Eri, R. D., Das, I., Lourie, R. and Florin, T. H. (2010) ER Stress and the Unfolded Protein Response in Intestinal Inflammation. Am. J. Physiol. Gastrointest. Liver Physiol. 25 Townsend, D. M., Manevich, Y., He, L., Xiong, Y., Bowers, R. R., Jr., Hutchens, S. and Tew, K. D. (2009) Nitrosative stress-induced s-glutathionylation of protein disulfide isomerase leads to activation of the unfolded protein response. Cancer. Res. 69, 76267634

26 Gotoh, T. and Mori, M. (2006) Nitric oxide and endoplasmic reticulum stress. Arterioscler. Thromb. Vasc. Biol. 26, 1439-1446

27 Herulf, M., Ljung, T., Hellstrom, P. M., Weitzberg, E. and Lundberg, J. O. (1998) Increased luminal nitric oxide in inflammatory bowel disease as shown with a novel minimally invasive method. Scand. J. Gastroenterol. 33, 164-169

28 Petrovic, V., Teng, S. and Piquette-Miller, M. (2007) Regulation of drug transporters during infection and inflammation. Mol. Interv. 7, 99-111

29 Stanislawowski, M., Wierzbicki, P. M., Golab, A., Adrych, K., Kartanowicz, D., Wypych, J., Godlewski, J., Smoczynski, M. and Kmiec, Z. (2009) Decreased Toll-like receptor-5 (TLR-5) expression in the mucosa of ulcerative colitis patients. J. Physiol. Pharmacol. 60 Suppl 4, 71-75

30 Sullivan, S., Alex, P. Dassopoulos, T., Zachos, N. C., lacobuzio-Donahue, C., Donowitz, M., Brant, S. R., Cuffari, C., Harris, M. L., Datta, L. W., Conklin, L., Chen, Y. and $\mathrm{Li}, \mathrm{X}$. (2009) Downregulation of sodium transporters and NHERF proteins in IBD patients and mouse colitis models. potential contributors to IBD-associated diarrhea. Inflamm. Bowel Dis. 15, 261-274

31 Thibault, R., De Coppet, P., Daly, K., Bourreille, A., Cuff, M., Bonnet, C., Mosnier, J. F., Galmiche, J. P., Shirazi-Beechey, S. and Segain, J. P. (2007) Down-regulation of the monocarboxylate transporter 1 is involved in butyrate deficiency during intestinal inflammation. Gastroenterology. 133, 1916-1927

32 Itzkowitz, S. H. and Yio, X. (2004) Inflammation and cancer IV. Colorectal cancer in inflammatory bowel disease: the role of inflammation. Am. J. Physiol. Gastrointest. Liver Physiol. 287, G7-17

33 Urquhart, B. L., Ware, J. A., Tirona, R. G., Ho, R. H., Leake, B. F., Schwarz, U. I., Zaher, H., Palandra, J., Gregor, J. C., Dresser, G. K. and Kim, R. B. (2008) Breast cancer 
resistance protein (ABCG2) and drug disposition: intestinal expression, polymorphisms and sulfasalazine as an in vivo probe. Pharmacogenet. Genomics. 18, 439-448

34 Zaher, H., Khan, A. A., Palandra, J., Brayman, T. G., Yu, L. and Ware, J. A. (2006) Breast cancer resistance protein (Bcrp/abcg2) is a major determinant of sulfasalazine absorption and elimination in the mouse. Mol. Pharm. 3, 55-61

35 Koelink, P. J., Mieremet-Ooms, M. A., Corver, W. E., Wolanin, K., Hommes, D. W., Lamers, C. B. and Verspaget, H. W. (2010) 5-aminosalicylic acid interferes in the cell cycle of colorectal cancer cells and induces cell death modes. Inflamm. Bowel Dis. 16, 379-389 36 Terdiman, J. P., Steinbuch, M., Blumentals, W. A., Ullman, T. A. and Rubin, D. T.

10 (2007) 5-Aminosalicylic acid therapy and the risk of colorectal cancer among patients with

11 inflammatory bowel disease. Inflamm. Bowel Dis. 13, 367-371 
2

3

4

5

6

7

8

9

10

11

12

13

14

15

16

17

20

21

22

23

24

25

26

27

28

29

30

31

32

33

34

35

36

37

38

39

40

41

42

43

44

45

46

47

48

49

50

\section{Figure legends}

Figure 1: Reduced ABCG2 expression in actively inflamed intestinal biopsies.

A) Representative images of intestinal biopsies IHC stained for ABCG2. Colon, Ctr) colon biopsy from healthy individual. Colon, IA) colon biopsy from an IBD patient in remission. Colon, A) colon biopsy from an IBD patient with active inflammation. Colon, ISC) colon biopsy from a patient with ischemic colitis. Colon, IFC) colon biopsy from a patient with infectious colitis. $S B$ Ctr) small bowel biopsy from a healthy individual. $S B$, IA) small bowel biopsy from a Crohn's disease patient in remission. SB, A) small bowel biopsy from a Crohn's disease patient with active inflammation. The ABCG2 expression is represented by red-brown staining on the apical membrane of IEC. Original magnification is 400x.

B) Decrease in the IEC fraction of colonic biopsies with ABCG2 expression during active inflammation. C) Actively inflamed small bowel displays reduced expression of ABCG2. B) and C) All intestinal biopsies were double blindly examined as negative, moderate or positive for ABCG2 membrane immune reactivity. Mann-Whitney $U$ tests and KruskalWallis tests were used for statistical analysis.

Figure 2: mRNA expression from intestinal biopsies.

A) No difference in ABCG2 mRNA expression in colonic biopsies. Relative mRNA (deltadeltaCt) expression corrected for Villin. Villin and ABCG2 are both IEC specific, thus ABCG2 mRNA is corrected for Villin to prevent underestimation in biopsies with less IEC. B) Increased pro-inflammatory cytokine expression in colonic biopsies from patients with active inflammation. Relative mRNA expression (deltadeltaCt) corrected for GapdH. IL8 and IL1 beta are pro-inflammatory cytokines and CHOP is an ER stress related gene. .

A) and B) Control are healthy individuals $(n=6)$, la IBD are biopsies with inactive inflammation $(n=8)$, a IBD are biopsies with active inflammation $(n=7)$. Mann-Whitney $U$ tests and Kruskal-Wallis tests were used for statistical analysis. C) XBP1 mRNA splicing. Agarose gel image of XBP1 splicing PCR products. Spliced XBP1 is an indicator for increased ER stress. $A$ is active IBD, $I A$ is inactive IBD and $H$ is healthy control.

Figure 3: Increased GRP78 expression in actively inflamed intestinal biopsies.

A) Representative images of intestinal biopsies IHC stained for GRP78. Colon, Ctr) colon biopsy from healthy individual. Colon, IA) colon biopsy from an IBD patient in remission. Colon, A) colon biopsy from an IBD patient with active inflammation. Colon, ISC) colon biopsy from a patient with ischemic colitis. Colon, IFC) colon biopsy from a patient with infectious colitis. $S B$ Ctr) small bowel biopsy from a healthy individual. $S B$, IA) small bowel biopsy from a Crohn's disease patient in remission. $S B, A)$ small bowel biopsy from a Crohn's disease patient with active inflammation. Expression of GRP78 is detected as redbrown colour and is present in the cytoplasm of IEC and mononuclear cells in the lamina propria. Original magnification is 400x. B) Increased in the IEC fraction of colonic biopsies with elevated GRP78 expression during active inflammation. C) Actively inflamed small bowel displays elevated GRP78 expression. B) and C) The biopsies were double blindly examined as positive, moderate or negative according the amount of positive GRP78 immune reactivity. Mann-Whitney $U$ tests and Kruskal-Wallis tests were used for statistical analysis.

Figure 4: Nitric oxide induced ER stress reduces ABCG2 protein expression and function. A) Western blot analysis showing the expression of GFP-ABCG2 protein using the antibodies BXP21 (anti-ABCG2) and anti-GFP. Tubulin (Tub) and beta-actin (b-Act) were used as loading control. MM is molecular marker. 
B) SNAP induces increasing levels of nitrite, a stable derivative of NO, in time.

2 Concentrations are measured from supernatants of HEK293t cells transiently transfected with GFP-ABCG2 (293t-AB cells).C) After transfection with GFP-ABCG2, HEK239t cells were stimulated with $0.625 \mathrm{mM}$ SNAP and XBP1 splicing was determined, and the ratio of un-spliced (U) and spliced (S) XBP1 were plotted. Ctr depict HEK239t cells only, 0 are

6 GFP-ABCG2 transfected cells without SNAP stimulation and other time points represents

7 GFP-ABCG2 transfected cells with $0.625 \mathrm{mM}$ SNAP. Gel picture is representative of three 8 independent experiments, graph represents the average of these three individual

9 experiments and error bars are SD.

10

Figure 5: Reduced ABCG2 activity and less membrane localization in ER stressed cells. Hoechst $33342(4 \mu \mathrm{M})$ DNA binding was measured to study the activity of ACBG2.

A) ABCG2 transfected cells were exposed to SNAP, tunicamycin or solvent for $16 \mathrm{~h}$ after which Hoechst 33343 binding was studied. See supporting Movies 1, 2 and 3. Original magnification 400x. B) The specific inhibitor of ABCG2, Ko143 (2 $\mu \mathrm{M})$ added 12 min after Hoechst $33342(4 \mu \mathrm{M})$ ), was used as a positive control for ABCG2 activity. The accompanying movie is supporting Movie 4. Original magnification 400x. C) 293t-AB cells were stained with ER-RFP. ER stress was induced by addition of SNAP or tunicamycin 16 19 h before ER-RFP staining.

20 All confocal images are representative of at least three independent experiments. Original 21 magnification 400x. 
Biochemical Journal Immediate Publication. Published on 25 Aug 2011 as manuscript BJ20111281

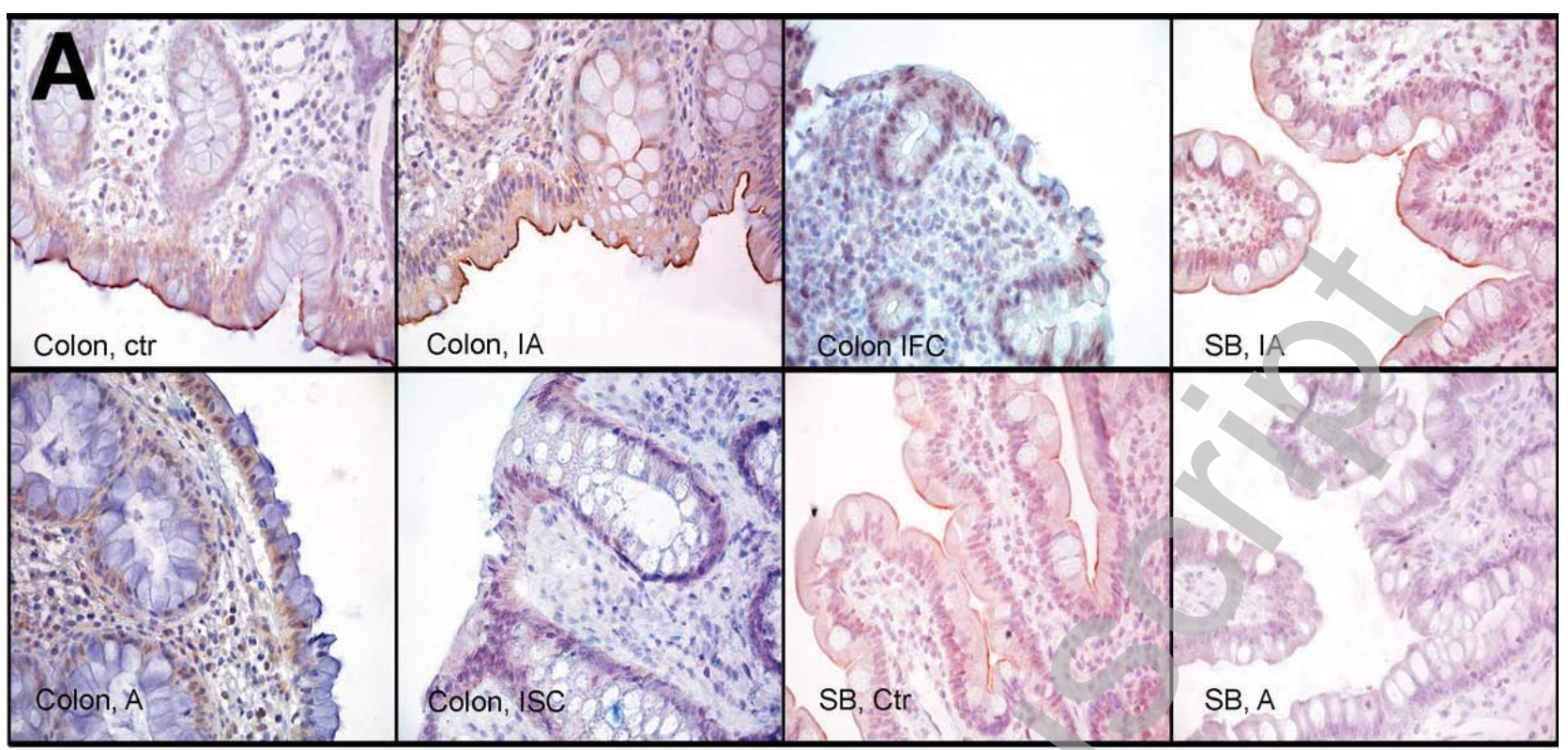

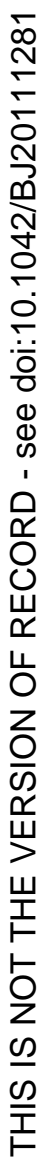
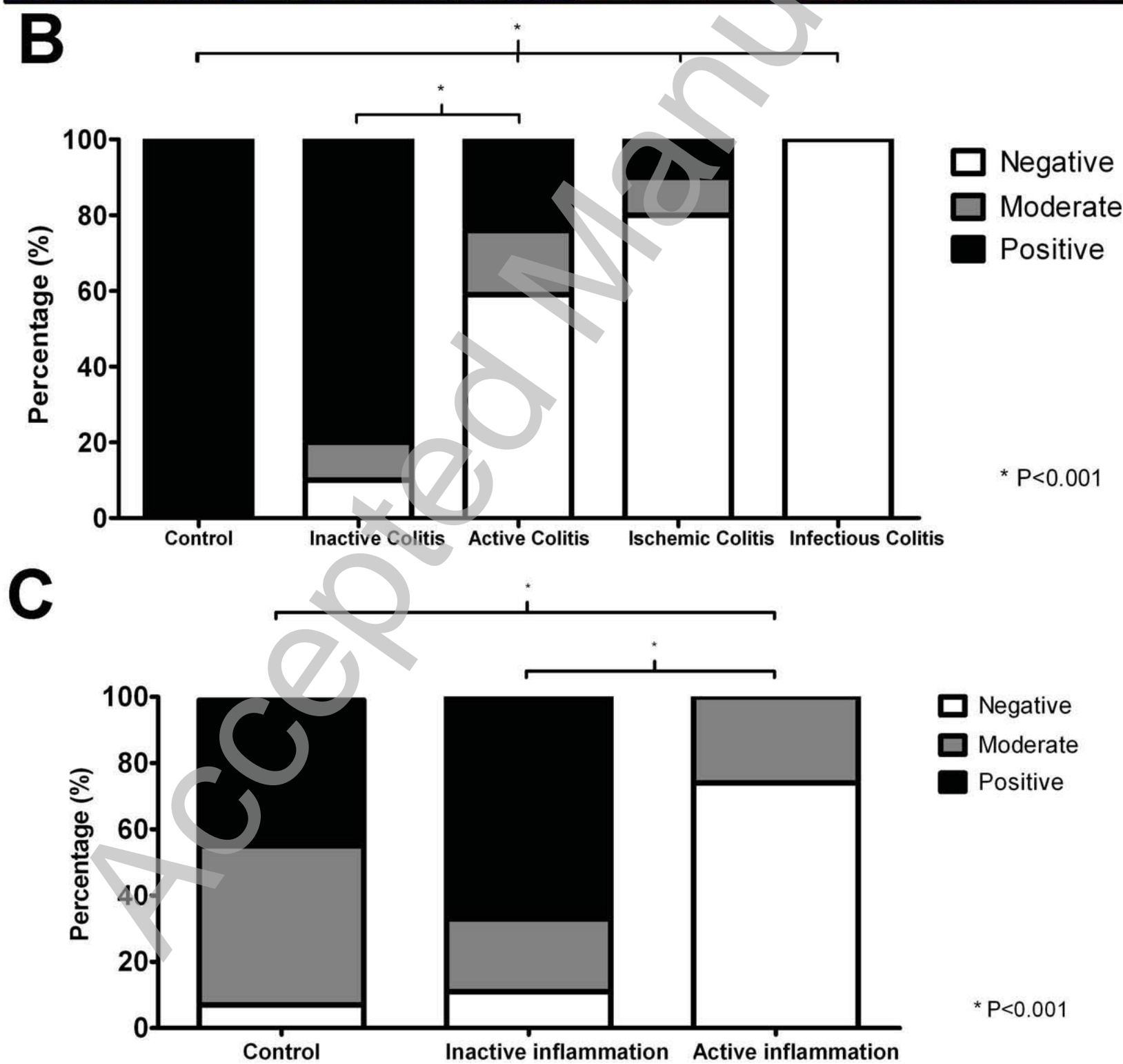

${ }^{*} \mathrm{P}<0.001$

Licenced copy. Copying is not permitted, except with prior permission and as allowed by law. (C) 2011 The Authors Journal compilation (c) 2011 Portland Press Limited 
A

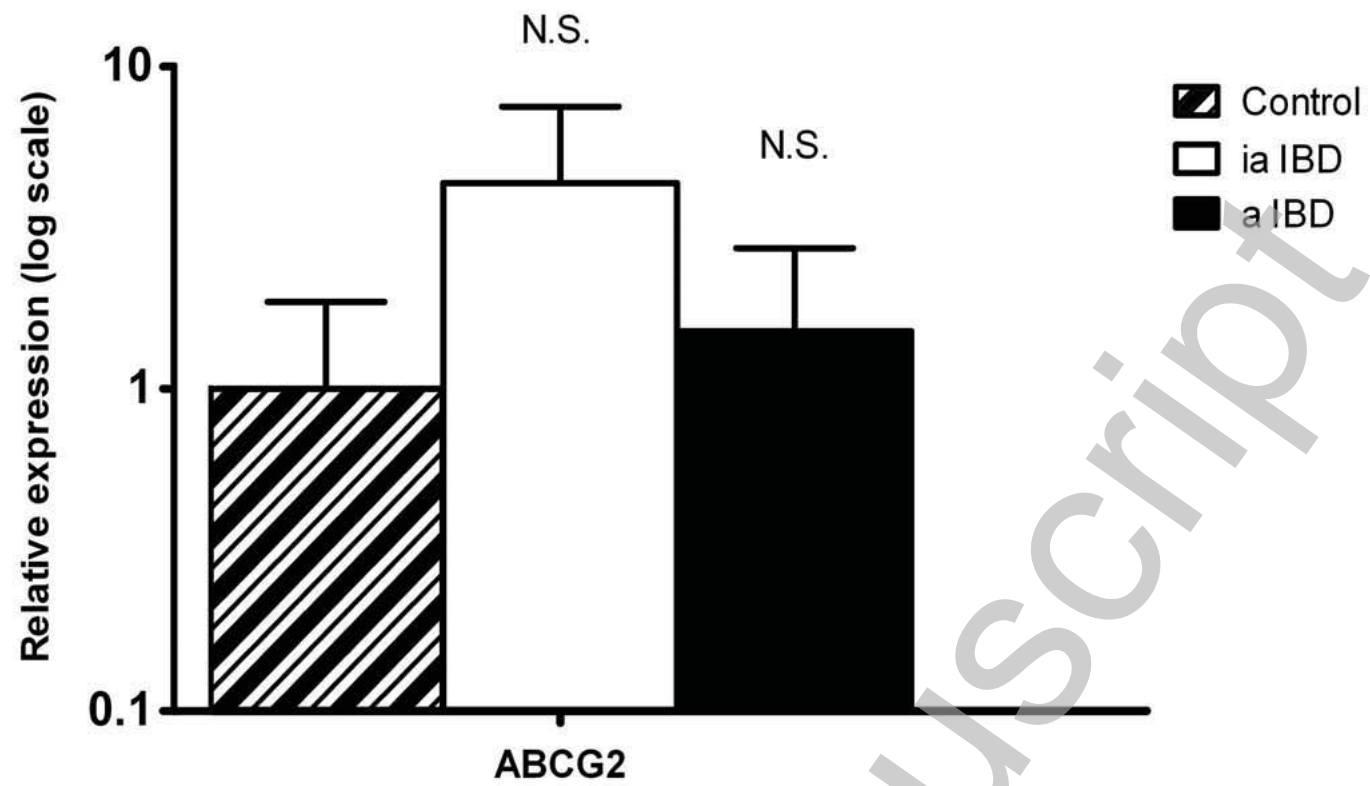

B

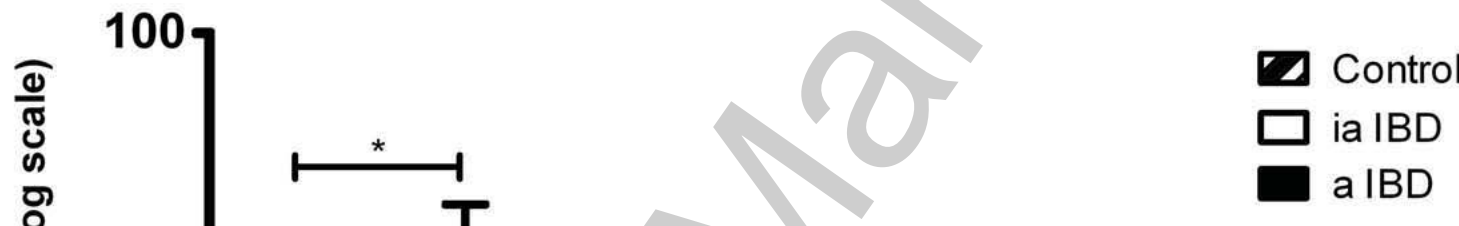

0

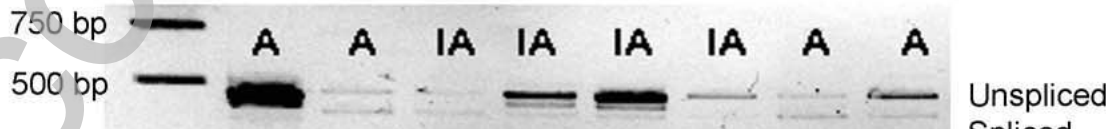

Spliced

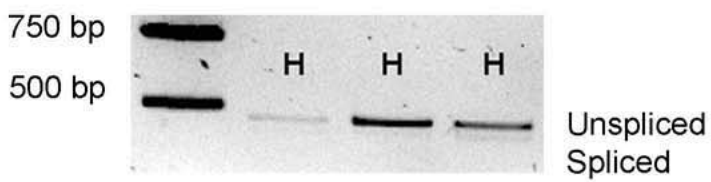

Licenced copy. Copying is not permitted, except with prior permission and as allowed by law. 
Biochemical Journal Immediate Publication. Published on 25 Aug 2011 as manuscript BJ20111281

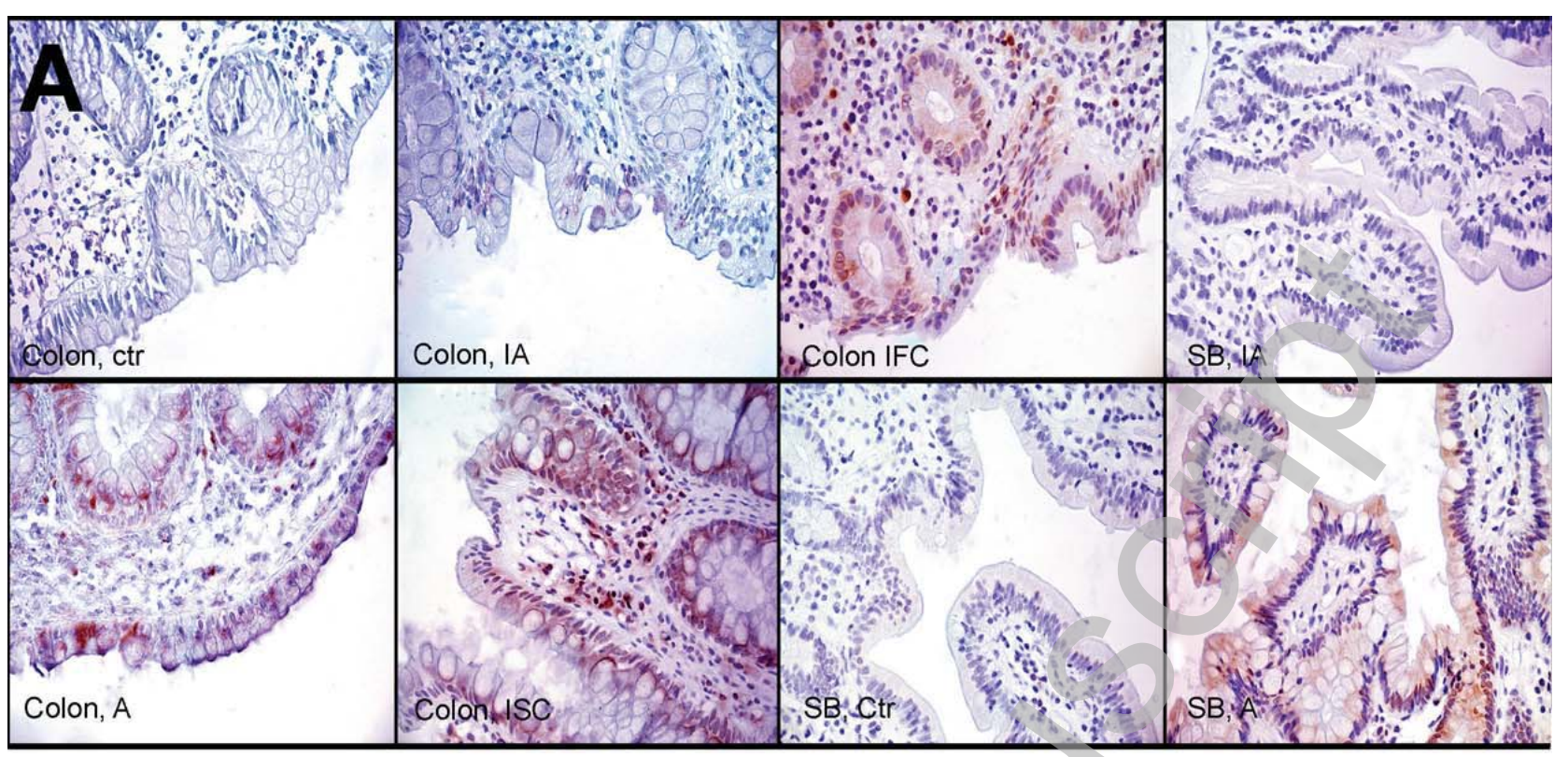

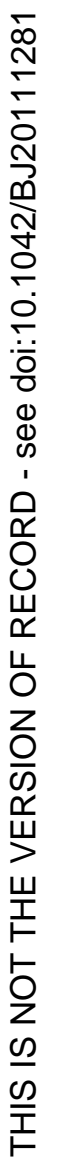

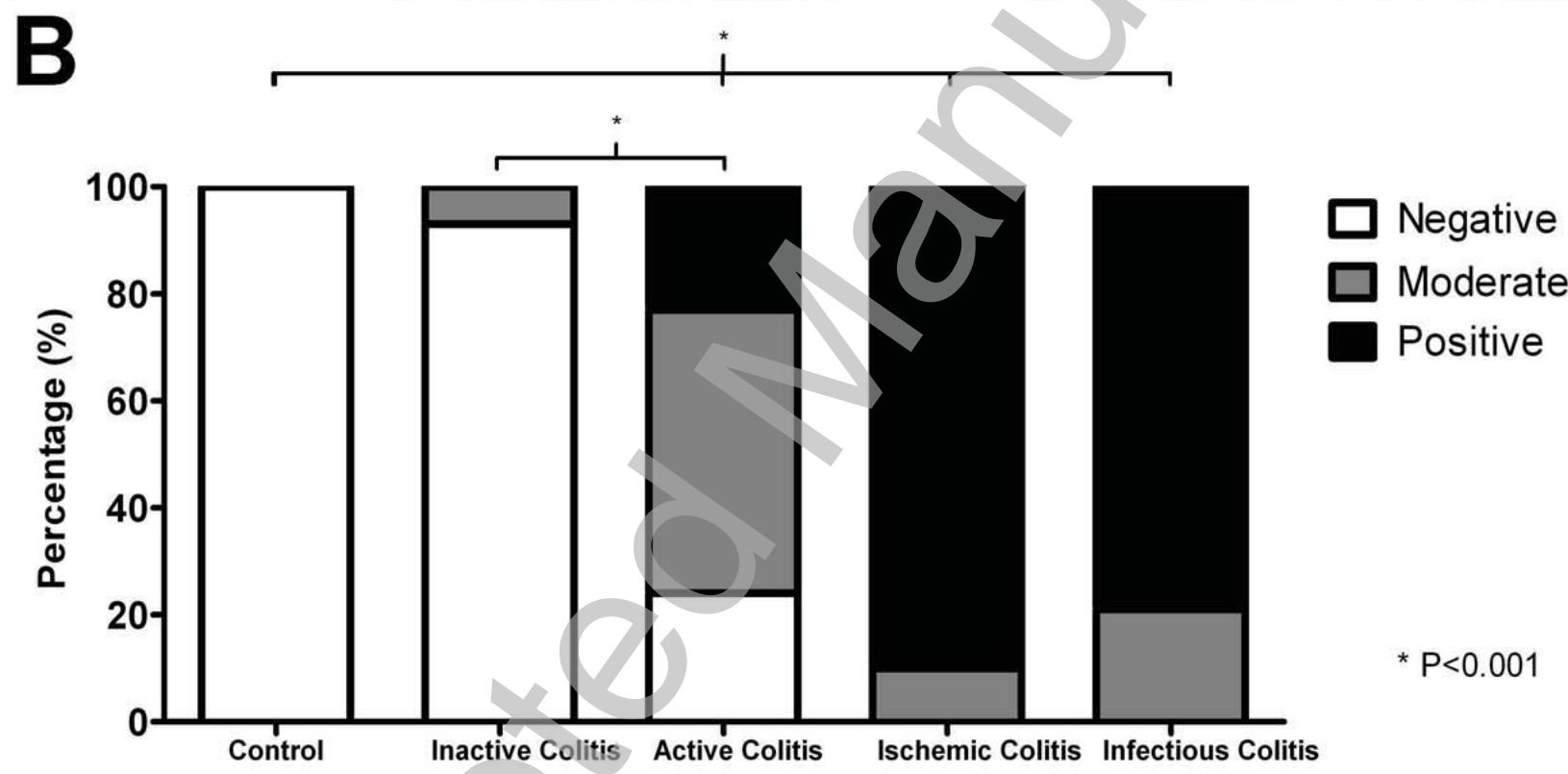

0

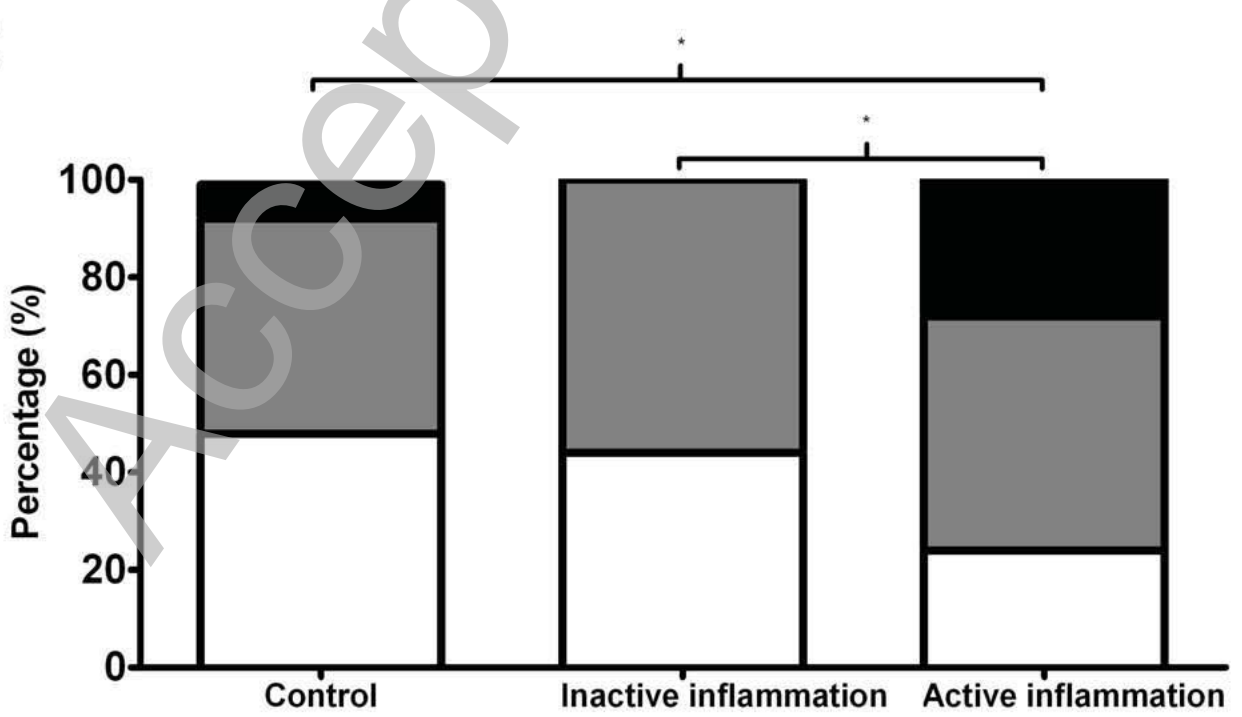

${ }^{*} \mathrm{P}<0.05$

Licenced copy. Copying is not permitted, except with prior permission and as allowed by law. (C) 2011 The Authors Journal compilation (c) 2011 Portland Press Limited 
